\title{
Benchmarking de modelos de negocio en empresas agrofrutícolas exportadoras: caso de empresas en Colombia y Chile'
}

Benchmarking of models of business in agricultural fruit export companies: Colombia and Chile case

Carlos F. Peña Alarcón* María del P. Fernández Rosas** Hernán M. Pinzón Mojica*** Diana C. Medina Hernández $z^{\star * *}$ Recibido: 29-08-2014 / Revisado: 10-09-2014 / Aceptado: 09-05-2015

\section{Resumen}

El objetivo del presente artículo es realizar la revisión teórica acerca de la aplicación del benchmarking en empresas del sector agrofrutícola y dar soporte, con base en la teoría, a los beneficios que se pueden lograr al efectuar dicho proceso. La metodología utilizada para este fin es la del estudio de caso y responde a las etapas del estudio de caso de Yin (2002). Este artículo de revisión es el producto de la investigación que está en curso.

1 Artículo de revisión.

* Administrador y Negociador Internacional, especialista en Gerencia de Mercadeo, candidato a Magíster en Negocios Internacionales, director del programa de Administración y Negocios Internacionales de la Universidad de Boyacá. carpena@uniboyaca.edu.co

** Magíster en Administración, docente de Administración y Negocios Internacionales, Universidad de Boyacá. mpfernandezr@uniboyaca.edu.co

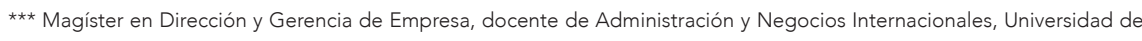
Boyacá.hmpinzon@uniboyacá.edu.co

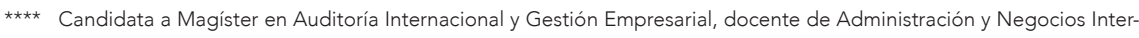
nacionales, Universidad de Boyacá. dcmedinah@uniboyaca.edu.co
Los resultados obtenidos del presente artículo muestran el benchmarking como una herramienta estratégica con la cual se busca establecer las mejores prácticas administrativas, tecnológicas y comerciales, con el fin de hacer más competitivas a las organizaciones con base en la comparación de una empresa con el líder del mercado, ya sea local, nacional o internacional.

El estudio de caso presenta el proceso de comparar a través del benchmarking el modelo de negocio agrofrutícola de una empresa chilena líder, frente al modelo de una empresa colombiana, mediante la identificación de los factores de competitividad que han permitido a la empresa chilena ubicarse como líder en el mercado. Estos factores de competitividad se determinan siguiendo las etapas del proceso 


\section{Abstract}

dadas en el modelo original de benchmarking, como son: planeación, análisis, integración y acción.

La investigación es no experimental cuantitativa, pues no se pretende manipular los fenómenos por estudiar ni sus variables. Se espera que los resultados constituyan un aporte significativo para el diseño de modelos de negocio agrofrutícolas competitivos y que se ajuste a las dinámicas nacionales del mercado de alimentos en Colombia.

Palabras clave: benchmarking, modelo de negocios, empresa agrofrutícola, factores de competitividad
This article reviews the theory about the application of benchmarking between agricultural fruit companies and to support, based on theory, the benefits obtained from this process. The methodology used is the case study and it responds to the stages of Yin's Case Study (2002). This article is the result of a current research. The results show to benchmarking as a strategic tool which seeks to establish the best administrative, technological and business practices toward more competitive organizations of the same sector; it consist in comparing an enterprise with the local, national or international leader of the market.

This article presents the compare process based on benchmarking methodology between business model of an agricultural fruit Chilean enterprise, as a market leader and a Colombian enterprise identifying the competitiveness factors that have enabled to the Chilean enterprise position as the leader in its market. Those competitiveness factors are determined following the stages of benchmarking original model, such as: planning, analysis, integration and action. The study is non-experimental quantitative and it does not pretend to manipulate neither the phenomena to study nor variables. The expectative is that the results of this study constitute a significant contribution to the design of competitive business models for agricultural fruit sector conforming national dynamics of food market in Colombia.

Keywords: benchmarking, business models, agricultural and fruit company, competitiveness factors. 


\section{Introducción}

El panorama agrario en Colombia es paradójico: por una parte, este es el segundo país en biodiversidad, cuenta con los cuatro pisos térmicos y es el cuarto país en extensión de tierra cultivable y el $38,3 \%$ de su potencial hídrico es utilizado en el riego de cultivos (Banco Mundial, 2009); pero, por la otra, tiene un nivel de concentración de la tierra del 0,847 según el índice de GINI (Rodríguez \& Cepeda, 2011), un desempleo rural del 6,6\% en condiciones de baja calidad, una tasa de migración campo-ciudad de 60 \% (Leibovich, Nigrinis, \& Ramos, 2008) y el 38,7 \% de los alimentos agrícolas que consumimos son importados (Promoción de Turismo, Inversión y Exportaciones -PROEXPORT-, 2012).

A finales de los ochenta el Departamento Nacional de Planeación inició una misión que tenía por objeto aumentar el conocimiento sobre la estructura y evolución de la agricultura colombiana desde los años cincuenta, sector que se consideraba estratégico para el desarrollo. El informe era una visión independiente y no oficial del sector agropecuario, de su evolución en las últimas décadas y de los problemas que impedían el mayor desarrollo del potencial sectorial. Este informe fue realizado en un momento crucial para el país, que se preparaba desde mediados de los ochenta para el proceso de apertura acentuado en 1990, año en que se presentó el informe al nuevo Gobierno de Gaviria; sin embargo, el Gobierno despreció el informe, pese a que era un instrumento muy valioso para regular el grado de apertura del sector agropecuario y evitar lanzarlo a un proceso muy traumático que, aunque necesario, podría haber sido más gradual y selectivo para dar tiempo a que el sector se preparara para afrontar una competencia internacional con prácticas comerciales desleales, de elevados subsidios y ayudas en los países más industrializados (Machado, 1999).

Adicionalmente, en Colombia poco se ha avanzado hacia la adopción de nuevos principios productivos, y, más bien, en los últimos años asistimos a un reforzamiento de un modelo económico basado fundamentalmente en la explotación de recursos naturales para la exportación, ya sea por medio de la gran producción agropecuaria de palma africana, la extracción minera de diversos materiales o la generación de energía hidroeléctrica mediante el desarrollo de megaproyectos (Zerda, 2011).

En este contexto de competencia de nuestro sector agrícola, se debe buscar la forma de tener mayores oportunidades para reactivar y profundizar en un mercado en el que Colombia tiene ventajas comparativas (Nash, 2011), siendo pertinente la identificación de un modelo de negocio agrícola a través del cual el país está, por una parte, supliendo su demanda interna de alimentos y, por otra, generando recursos con la exportación de los mismos. 
Se expone el caso de Chile, en donde la fruticultura, la industria forestal y la agroindustria se adecúan a las reformas y ajustes y a las nuevas condiciones de apertura y se insertan con dinamismo en el sector exportador. Los sectores frutícola y forestal, que en conjunto representan dos tercios de las exportaciones silvoagropecuarias, son símbolos exitosos del modelo exportador chileno (Portilla, 2000).

A través del benchmarking se muestra la comparación del modelo agrofrutícola colombiano con el chileno, y se espera así dar un aporte a la literatura que ha profundizado en el mejoramiento del sector en Colombia y constituye una base para nuevos estudios que permitan detectar y aplicar herramientas eficaces que faciliten la generación de estrategias en búsqueda del mejoramiento y la competitividad.

\section{Revisión de literatura}

Se presenta la importancia del benchmarking como herramienta para la comparación y búsqueda de las mejores prácticas que desarrollan empresas líderes del sector en estudio para luego aplicarlas en su propia industria (Arrieta, Botero, \& Maria, 2010; Spendolini, 2005). El benchmarking como herramienta administrativa proporciona conocimientos necesarios para ayudar a comprender cómo una empresa líder se compara con otras organizaciones para destacar los procesos que conllevan la competitividad y de esta manera diseñar y aplicar nuevos modelos en el interior de la organización (Anand \& Kodali, 2008; Combita, 2011; Dembowski, 2013), dando claridad en cuanto al proceso como determinante de qué, cómo y con quién se va a medir (Boxwell, 1995).

Diferentes casos de éxito en la aplicación del benchmarking son utilizados para mostrar los beneficios del estudio, se resalta el caso de Xerox como pionero en benchmarking (Rico \& Irace, 1996; Agredano, 2005; Cámara Oficial de Comercio, Industria e Navegación de Santiago de Compostela, 2003; Solfa, 2012; ADERASA, 2012; Cámara Colombiana de la Infraestructura, 2012). Por lo anterior, se determina que la implementación del benchmarking ha contribuido a identificar diferentes modelos de negocio, lo que permite optar por el más idóneo o que arroje las mejores prácticas, que se aplicarán a su vez en otro determinado sector (Abbas \& Mann, 2010).

En este artículo se toman como base los pasos o etapas del proceso de benchmarking del modelo original realizado en Xerox (Camp, 1989; Gutiérrez, 2005), por ser el más citado en la literatura especializada y el más seguido por las empresas que han realizado benchmarking en sus organizaciones (Anand \& Kodali, 2008).

Se mencionan los diferentes modelos de negocio que permiten enmarcar las formas de hacer negocios, sin especificar una en particular que sea para el sec- 
tor agrícola, a fin de determinar los factores de éxito orientados hacia la creación de valor y sostenibilidad en mercados altamente competitivos para las empresas (Afuah \& Tucci, 2001; Johnson, Christensen \& Kagermann, 2008) que sean capaces de cambiar la forma de hacer las cosas para convertirse en un estándar de nuevas generaciones de empresarios (Magretta, 2002, p. 4).

El concepto de modelo de negocio es objeto de análisis y de reflexión sobre las nuevas formas de generar ingresos en las empresas que principalmente se fundamentan en el desarrollo tecnológico (Zott, Amit, \& Massa, 2010), siendo el modelo de negocio la forma en que la empresa puede permanecer en el tiempo y generar ingresos, soportada en la relación con sus clientes (Rappa, 2004, p. 34). El modelo de negocio se puede definir como el conjunto de actividades que le permite seleccionar un mercado objetivo, los productos y sus características para ofrecer en el mercado con un aspecto diferenciador (Drucker, 1986). Además de la estructura del modelo se tiene en cuenta el gobierno de las transacciones que se da en el proceso negociador, las cuales están orientadas hacia la creación de valor mediante la maximización de las oportunidades del negocio (Amit \& Zott, 2001). De igual forma, se plantea la forma en que las empresas, a través de una estrategia clara, generan utilidades, tienen disponibilidad de recursos e identifican los procesos que generan valor al cliente (Cassadesus-Masanell \& Ricart, 2010, p. 195).

\section{Enfoque metodológico}

Este artículo permite determinar de qué manera los modelos de negocio de las empresas del sector agrícola, particularmente de productores y exportadores de frutas de Chile y Colombia, pueden ser comparados a través de la herramienta del benchmarking, con el fin de identificar las mejores prácticas y aplicarlas a la empresa que busca las mejoras. El enfoque de lo particular hacia lo general, al igual que el estudio de carácter cualitativo que se quiere llevar a cabo, conllevan la adopción de un enfoque epistemológico inductivo (Hernández, Fernández, \& Baptista, 2010).

Teniendo en cuenta que se toman dos empresas para desarrollar el benchmarking, se establece la metodología del estudio de caso de Yin (Yin, 2002).

\section{Estudio de caso}

Teoría preliminar y unidades de análisis

Las tendencias mundiales del consumo de frutas han mostrado un crecimiento importante en el consumo per cápita de frutas y hortalizas a finales del siglo pasado. El valor del comercio mundial hortofrutícola creció 3,6 \% anual promedio entre 1990 y el año 2000, al pasar de 62.919 millones de dólares en 1990 a 82.407 millones de dólares en el año 2000. En este mismo período el valor del comercio exclusivamente de frutas creció 3,5 $\%$ anual promedio y pasó de 18.556 millones de dólares 
a 28.528 millones de dólares en 1996, año a partir del cual empieza a decrecer hasta llegar a 24.778 en el 2000. Durante el primer quinquenio de los noventa, la tasa de crecimiento anual promedio fue de $6,4 \%$, mientras que durante la segunda mitad de la década fue del 3,5\% anual promedio. Durante este mismo período, las frutas tropicales fueron las que mayor participación tuvieron en el valor del comercio mundial de frutas con el $27 \%$. Le siguieron en importancia los cítricos con un $20 \%$, los caducifolios (manzana, durazno, ciruela, pera, etc.) con 19 $\%$, las nueces con $13 \%$, y las uvas, $10 \%$. Los grupos de productos cuyo valor en el comercio creció por encima del promedio, fueron justamente las frutas denominadas tropicales, con una tasa de crecimiento anual promedio de $6 \%$, seguidos por las uvas, 5,3\%, y las bayas, 4,7\%. Los demás grupos, que representan el $58 \%$ del valor del comercio (caducifolios, cítricos, nueces y otros), crecen alrededor del 2,4 \% anual promedio (Hernández \& Pinzón, 2004). Además de ser la categoría más representativa en el comercio de frutas frescas, las frutas tropicales presentan las tasas de crecimiento más altas, sin embargo algunas frutas han venido disminuyendo su ritmo de crecimiento como el banano cuya tasa promedio anual pasó de 6,3\% entre 1993 y 1997 a una tasa negativa de 3,5\% entre 1998 y el 2002, lo mismo ocurrió con el melón donde la tasa promedio anual pasó de 7,7\% a - 0,7\% en el mismo periodo. Para algunos expertos lo anterior se explica por el hecho que estos mercados han llegado a una fase de madurez (Corporación Colombia Internacional Ministerio de Agricultura y Desarrollo Rural, 2002).
De igual forma, entre las tendencias del consumo es interesante resaltar como recientemente se ha extendido en Estados Unidos y en Europa lo que se conoce como la dieta low carb o de disminución en el consumo de calorías y aumento en el de proteínas, cuyo principio fundamental es reducir el consumo de carbohidratos, la principal fuente de energía del organismo, de modo que este se vea obligado a gastar las reservas de grasa del cuerpo, provocando la disminución de peso. Es tal la importancia de esta tendencia, que el bajo contenido de calorías empieza a contarse entre las características que más valora el consumidor en los alimentos (Organización de las Naciones Unidas para la Agricultura y la Alimentación, 2002).

El comercio de frutas se encuentra concentrado en las regiones de ingresos más altos: la Unión Europea, con el 50 \%, Estados Unidos, con el 12,5 \% y Japón, con el $7 \%$, y se distribuye por categorías de la siguiente manera: frutas frescas $(30,6 \%)$, procesadas $(30,3 \%)$ y jugos de frutas (Organización de las Naciones Unidas para la Agricultura y la Alimentación, 2002).

Según el Plan Frutícula Nacional (PFN), el registro mundial de frutas presenta una tendencia creciente que se puede explicar por los cambios en los ingresos económicos, las estructuras poblacionales y por la tendencia a creer más en las propiedades nutricionales y funcionales de las frutas (Legiscomex, 2013). 
Las importaciones de frutas en Colombia están concentradas en manzanas y peras, que representaron el 54.8 \% del total de la compra de frutas externas en 2001; en el primer caso, el país importó 50.533 toneladas por un valor FOB de US\$21.6 millones, mientras que en el caso de las peras el volumen importado fue de 14.042 toneladas por valor FOB de US\$ 6.5 millones para el mismo año, lo que permite entrever la potencialidad de Colombia en cuanto a la producción y consumo de frutas se refiere.

En Colombia se ha contado con un nivel de producción destinado principalmente al consumo interno y ha tenido que acudir a importaciones que satisfagan parte del mercado interno. La producción con destino a la exportación se ha concentrado principalmente en el banano, cuya tasa exportadora en 2001 alcanzó el $95.4 \%$ del volumen producido, otras frutas como la uchuva, pitaya e higos se registraron con una tasa exportadora entre el 20 y 30 \% (Hernández \& Pinzón, 2004). En el 2011, Colombia registró una producción de frutas de 3,4 millones de toneladas, un $4 \%$ más que en el 2010 cuando fueron 3,1 millones de toneladas, según últimas cifras suministradas por el Ministerio de Agricultura y Desarrollo Rural (Legiscomex, 2013). En este mismo año 2011, se apreció un dinamismo en el sector de las frutas cuyo crecimiento fue de 3,7 \%, tasa mucho mayor a la que en general presentó el sector agrícola de 1,3\%. En términos de volumen de producción para el año 2013, las principales líneas productivas fueron: banano, cítricos, piña, mango, aguacate y naranja (Asohofrucol, 2013). Se reportó un aumento en las siguientes frutas en diferentes mercados de exportación: uchuva, gulupa, granadilla, pitahaya, tomate de árbol, maracuyá, feijoa, chirimoya, arándonos, mango (Legiscomex, 2013).

Aunque se puede percibir el potencial y variedad que la producción colombiana puede ofrecer, no se presentan en las estadísticas productos que hayan logrado alcanzar una alta tasa exportadora como la ha desarrollado el producto insignia de exportación que es el banano o tasas de crecimiento constantes en el paso de los años.

Lo que se busca entonces es poder ofrecer una forma de aprovechamiento de ese potencial que tiene el sector frutícola en Colombia, mediante el conocimiento de las mejores prácticas del país que se ha considerado el mejor en este sector y ha logrado mantener su tasa exportadora en constante crecimiento, identificando sus fortalezas y estrategias a fin de presentarlas al sector colombiano, para que adapte e implemente aquellas que hasta el momento no ha desarrollado y, de esta forma, llegue a ser reconocido como líder exportador.

Adicionalmente, las perspectivas de crecimiento del sector son bastante alentadoras, considerando las tendencias positivas en la producción, debido a la creciente demanda de productos hortifrutícolas a nivel 
internacional, como resultado de la mejora en los hábitos de consumo saludables. De igual forma, el auge en los últimos años de la firma y entrada en vigencia de acuerdos comerciales con otros países, ha logrado una reducción arancelaria sustancial de los productos hortifrutícolas que, sin, duda generará un dinamismo interesante para el sector, reflejado en nuevas y mejores oportunidades. En este contexto Asohofrucol, con recursos del Fondo Nacional de Fomento Hortifrutícola, ha identificado alternativas de mejora y fomento para los productores mediante planes, programas y proyectos como el Plan Nacional de Fomento Hortifrutícola y los planes de negocios desarrollados conjuntamente con el Programa de Transformación Productiva -PTP- (Asohofrucol, 2013). De esta forma, junto con el PTP, se privilegiaron siete líneas sobre las cuales se van a dirigir actividades en pro del incremento de las exportaciones de aguacate Hass, mango, fresa, piña, papaya, cebolla de bulbo y ají.

Las proyecciones cuantitativas a 2030 son las siguientes:

En total, se prevé aumentar 110.000 hectáreas en estos siete productos, las cuales generarán alrededor de 133.000 nuevos empleos directos y, en ese mismo sentido, crecerán las exportaciones tanto en fresco como procesado en aproximadamente 637.000 toneladas (Asohofrucol, 2013). Entre las frutas exóticas más exportadas por Colombia se encuentran la uchuva, la gulupa, la granadilla y la pitahaya. Por su parte, la prin- cipal empresa exportadora de este tipo de productos es Ocati S.A, seguida por Novacampo S.A., C.I. Caribbean Exotics S.A., y C.I. y Andes Export Company S.A., entre otras. (Legiscomex, 2013)

En el 2012, Colombia exportó más de USD 48,6 millones de frutas exóticas, de los cuales Ocati S.A. participó con USD 11, 099 millones en exportaciones de uchuva, gulupa, granadilla, pitahaya, tomate de árbol, maracuyá y feijoa. De lo anterior se deduce que Ocati S.A. tomó parte en un $23 \%$ del total de exportaciones de frutas exóticas en Colombia (Legiscomex, 2013).

Ocati S.A. es una empresa colombiana, creada hace 24 años, pionera y líder en la producción y exportación de frutas y vegetales exóticos. Está comprometida en la producción de frutas de primera calidad, y para ello cuenta con un equipo de agrónomos que visitan continuamente las áreas de producción ubicadas en una variedad de condiciones climáticas en Colombia. El equipo trabaja en estrecha colaboración con los empleados en el campo, así como con los productores, con el fin de cumplir con las normas GlobalGAP. Para asegurar la calidad de sus productos, Ocati S.A., después de cosechar la fruta, la lleva a HACCP-IFS, casa de embalaje certificada, ubicada en el municipio de Chía, a unos 40 minutos al norte de Bogotá. (Ocati S.A., 2014). 
Ocati S.A. cuenta con un amplio portafolio de frutas como baby banana, baby mango, cacao fruit, cactus figs, curuba, feijoa, granadilla, guanábana, guayaba, lulo, mamoncillo, maracuya, pepino melón, Physalis (aguaymanto o uchuva), pitahaya, Purple Passion Fruit y tamarillo cuyos principales mercados destino de exportación son: Países Bajos, Alemania, Francia, Hong Kong y Costa Rica (Legiscomex, 2013).

Por lo anterior, se seleccionó a Ocati S.A. como la empresa colombiana más competitiva en el mercado exterior en producción y comercialización de frutas exóticas y que por las características expuestas es la apropiada para involucrar en el proceso de benchmarking.

\section{Caracterización del sector agrícola en Chile}

Chile en la actualidad es uno de los principales productores y exportadores de fruta en Latinoamérica y en el mundo. La industria frutícola en Chile es bastante extensa, tanto en su nivel de producción como en su nivel de exportación.

La industria frutícola en Chile cuenta con más de 7800 productores de fruta. Las frutas que tienen mayores volúmenes de producción son las uvas de mesa, las manzanas y las paltas, para un total de 136.000 hectáreas dedicadas a la producción de estos productos. Se destaca además que Chile dispone de 310.266 hectá- reas para la producción de frutas (Asociación Chilena de Fruta Fresca, 2012).

En cuanto al rubro de exportaciones, es una industria que tiene 747 compañías exportadoras y más de 100 países en el mundo importan fruta chilena de 75 clases de frutas diferentes.

En su estructura comercial, el $65 \%$ de la producción total se exporta y, por si fuera, poco sus volúmenes de exportación son de 2.394.000 toneladas en el año 2008, 2.466.000 en el 2009, 2.648.000 en el 2010, y 2.591.000 en el 2011 (Asociación Chilena de Fruta Fresca, 2012).

Comparando las cifras a nivel mundial, Chile se muestra como el líder en exportaciones dentro del hemisferio sur, representando el 59,3\% de las exportaciones de esta zona geográfica, dentro de las cuales se encuentran uvas, manzanas, kiwis, paltas, ciruelas, duraznos, peras, ciruelas y arándanos.

Por otro lado, según el reporte de fruitsfromchile, en frutas como uvas, ciruelas, manzanas, arándanos, duraznos y paltas, Chile se posiciona como el líder mundial en la exportación de estos productos (Asociación Chilena de Fruta Fresca, 2012). Los principales destinos de exportación de las frutas chilenas son Estados Unidos con un 38 \% de participación, Europa con un $29 \%$, Asia $11 \%$, Latinoamérica $16 \%$, y el Medio Oriente con el $6 \%$. La configuración de la industria 
frutícola en Chile hace que sea referente mundial para observar, analizar y adoptar sus principios, estrategias y prácticas empresariales por su nivel de productividad y por su volumen de exportaciones.

En un informe de la reconocida página Portal Frutícola de Chile, (Portal Frutícola, 2010) se reveló detalladamente la composición y ranking de las principales empresas exportadoras de frutas en Chile (Portal Fruticola, 2010). El informe relata que la empresa líder es Standard Dole sede Chile, la cual para el 2010 alcanzó a exportar 149 millones de kilos de fruta, seguido de la Transnacional Unifrutti, que exportó para este año 121, 5 millones de kilos; así mismo le sigue la empresa David el Curto con un volumen de exportaciones de 104 millones de kilos de fruta exportados y por último se encuentra la empresa Copefruto con 98 millones de kilos.

Sin embargo, la página principal de la Sociedad Agrícola de Bio Bio AG relata que el ranking de exportadores chilenos presentó un cambio entre el 2011 y el 2012 (Socabio AG, 2013).

Para el año 2012 se informa que las multinacionales Standard Dole y Unifrutti siguen asumiendo el primer y segundo lugar respectivamente. El cambio se dio a partir de la tercera posición que ahora fue asumida por la empresa Frusan, que pasó de exportar 94,4 millones de kilos en el 2011 a 110, 4 millones de kilos de fruta en el año 2012.
En este sentido, aunque existe un amplio número de empresas grandes con las cuales la industria nacional frutícola colombiana podría compararse para adquirir mejores prácticas en todo sentido, sería recomendable gestionar un benchmarking en primera instancia con la empresa Standard Dole por ser la líder en exportaciones de fruta chilena. (Socabio AG, 2013).

\section{Etapas de benchmarking}

El benchmarking que se desea llevar a cabo consta de una serie de pasos para su implementación y aunque la literatura no presenta un número unánime de pasos, en el presente artículo nos guiaremos del modelo original implementado en Xerox ${ }^{\circledR}$ (Camp, 1989), ya que es el más citado dentro de la literatura especializada y el más seguido por las empresas que han realizado benchmarking en sus organizaciones (Anand \& Kodali, 2008). Dicho modelo consta de cuatro etapas: planeación, análisis, integración y acción.

\section{Etapa 1. Planeación}

Con el fin de determinar las mejores prácticas de la empresa líder chilena, se establece que es necesario realizar un benchmarking de tipo interno a las empresas seleccionadas como unidades de análisis, es decir, para la empresa Ocati S.A. en Colombia y para Standard Dole en Chile. 
Las fuentes para la obtención de esta información son las empresas seleccionadas y las entidades públicas y privadas relacionadas con las actividades que evalúa cada factor y que provean o consoliden la información relacionada con cada uno. El método de recolección de datos es a través de encuestas. Se hacen contactos directos personalmente o vía internet con el fin de generar confianza en las empresas seleccionadas y contar con su colaboración para la realización del estudio.

\section{Etapa 2. Análisis}

Con base en la información recolectada se inicia su organización y análisis para establecer las brechas o procesos en los que existen fuertes diferencias entre Ocati S.A. y Standard Dole. A través de este análisis se espera determinar las mejores prácticas con las que se desarrolla la siguiente etapa.

\section{Etapa 3. Integración}

El diagnóstico realizado debe conllevar la realización del plan de acción en el interior de la empresa Ocati S.A., de tal forma que se puedan integrar las mejoras necesarias provenientes de las "mejores prácticas" aplicadas por Standard Dole para ganar competitividad en el sector.

\section{Etapa 4. Acción}

En esta etapa se proponen las mejoras mediante la presentación de planes estratégicos a la organización. En esta etapa la "acción" corresponde a la entrega de planes diseñados que estén apropiadamente adecuados para la implementación en la compañía, sin embargo no se antepone la suposición de que la empresa decida implementarlos, por lo tanto el aporte se limita a la entrega de los planes resultados de este estudio. Este etapa, más que ser el fin del proceso es, idealmente, el inicio de un nuevo proceso en busca del mejoramiento continuo de la organización (Gutiérrez, 2005).

\section{Modelos de negocio}

El concepto de modelo de negocio es objeto de análisis y de reflexión sobre las nuevas formas de generar ingresos en las empresas que principalmente se fundamentan en el desarrollo tecnológico (Zott, Amit, \& Massa, 2010), siendo el modelo de negocio la forma en que la empresa puede permanecer en el tiempo y genera ingresos, soportada en la relación con sus clientes (Rappa, 2004, p. 34). El modelo de negocio se puede definir como el conjunto de actividades que le permite seleccionar un mercado objetivo, los productos y sus características para ofrecer en el mercado, con un aspecto diferenciador (Slywotzky, 1996, p. 4), (Timmers, 1998, p. 2), (Drucker, 1986), (Morris, Schindehutte \& Allen, 2005, p. 727). Con el desarrollo de un 
estudio de benchmarking que identifique los factores de éxito en la competitividad de las unidades de análisis, se podrá identificar el modelo de negocio que, según las definiciones anteriores, es el que se lleva a cabo para permanecer en el tiempo y generar los ingresos que han permitido situarse como líder en el mercado. Los factores de la competitividad que serán objeto de benchmarking facilitarán la identificación del modelo de negocios de Standard Dole. Lo anterior es de gran importancia dada la falta de identificación de modelos específicamente utilizados en el sector agrofrutícola y permitirá ofrecer los planes estratégicos para la compañía Ocati S.A. en Colombia, que se espera sea de gran aporte para su crecimiento y mejora competitiva.

\section{Conclusiones}

El benchmarking es una herramienta muy valiosa para la identificación de los factores de competitividad que hacen que una compañía se destaque como la de mejores prácticas en su sector y, así mismo, facilita el mejoramiento de la competitividad cuando estas prácticas son adoptadas por otras compañías.

Las exportaciones del sector agrofrutícola en Colombia muestran una gran expectativa de crecimiento debido a la creciente demanda como resultado de la mejora de los hábitos de consumo, lo que representa una oportunidad importante de crecimiento, para lo cual aplicarán de manera positiva la identificación de me- jores prácticas de una empresa líder en la exportación ubicada en el mercado chileno.

Los factores de la competitividad determinados para el benchmarking permiten hacer una comparación de variables a nivel interno y externo de las compañías, teniendo en cuenta que ambos contextos son igualmente importantes para determinar el origen de las mejores prácticas. 


\section{Referencias}

Abbas, A. \& Mann, R. (2010). An invetsigation of the adoption and implementation of benchmarking. E-Bussines and Operation Management.

Afuah, A. \& Tucci, C. (2001). Internet business models and strategies: text and cases. Irvin: Mc Graw Hill.

Agredano, R. (2005). The paradigmate. Recuperado de http://www.theparadigmagate.com/espanol/mediacenter/publicaciones/Benchmarking_como_Alternativa_para_Mejorar_Procesos.pdf

Amit, R. \& Zott, C. (2001). Value creation in e-business. Strategic Management Journal, 22, 493-520.

Anand, G. \& Kodali, R. (2008). Benchmarking the benchmarking models. Benchmarking: An International Journal, 15 (3), 257-291.

Arrieta, J., Botero, V. \& Maria, R. (2010). Benchmarking about lean manufacturing in the textile sector in Medellin. Journal of Economics, Finance \& Administrative Science, 15 (28), 15-35.

Asociación Chilena de Fruta Fresca. (2012). Fruits from Chile. Recuperado de http://www.fruitsfromchile.com/esp/statistics.php

Asociación Chilena de Fruta Fresca. (2012). Industria Frutícola Chilena. Santiago de Chile: Asociación Chilena de Fruta. 
Asociación de Entes Reguladores de Agua Potable y Saneamiento de las Américas -Aderasa-. (2012). Asociación de Entes Reguladores de Agua Potable y Saneamiento de las Américas. Buenos Aires: Grupo Regional de Trabajo.

Asohofrucol. (2013). Balance y perspectivas del sector hortifrutícola. Revista Frutas y Hortalizas, 11-14.

Banco Mundial. (2009, 13 de abril). Indicadores Banco Mundial. Recuperado de: http://datos.bancomundial.org/indicador/AG.LND.AGRI.ZS/countries

Boxwell, R. (1995). Benchmarking para competir con ventaja. España: McGraw Hill.

Cámara Colombiana de la Infraestructura. (2012, mayo). Presentaciones. Recuperado de http://infraestructura.org.co/presentaciones/evconsultoria/HVELANDIA.pdf

Cámara Oficial de Comercio, Industria y Navegación de Santiago de Compostela. (2003, 20 de octubre). Cámara Comercial de Compostela. Recuperado de http://www.camaracompostela.com: http://www.camaracompostela.com/bench/manual.pdf

Camp, C. (1989). Benchmarking: The search the industry best practices that lead to superior performance. Milwaukee: Quality Press. 
Cassadesus-Masanell, R. \& Ricart, J. (2010). From strategy to business model and to tactics. Long Range Planning, 43, 195-215.

Combita, L. (2011). Benchmarking de programas académicos de posgrado en administración y negocios de universidades en Estados Unidos y Latinoamérica, y los programas académicos de la Universidad EAN. Bogota, Colombia: EAN.

Corporación Colombia Internacional Ministerio de Agricultura y Desarrollo Rural. (2002). Comercialización y encadenamientos productivos en el sector agroalimentario. Bogotá: Corporación Colombia Internacional.

Cuadernos de Contabilidad. (2012). Analisis de las implicaciones no financieras de la aplicación de las NIIF para pymes en las medianas entidades en Colombia. Cuadernos de contabilidad, 12.

Dembowski, F. (2013). The roles of benchmarking, best practices \& innovation in organization effectiveness. The International Journal of Organizational Innovation, 5 (3).

Drucker, P. (1986). La innovación y el empresario innovador: La práctica y los principios. Buenos Aires: Sudamericana, Norma.

Fundación IFRS. (2009). Material de formación Normas Internacionales de Informacion Financiera para pymes. Londres: Consejo de Normas Internacionales de Contabilidad. 
Gutiérrez, H. (2005). Calidad total y productividad. México: Mc Graw-Hill Interamericana.

Hernández, R., Fernández, C. C. \& Baptista, L. P. (2010). Metodología de la investigación (5 ed.). México: McGraw Hill.

Hernández, R. \& Pinzón, M. A. (2004). Perfil competitivo del sector frutícola colombiano: las frutas como alternativa de crecimiento económico. Bogotá: Universidad de San Buenaventura.

Legiscomex. (2013). Inteligencia de mercados. Exportación de frutas exóticas colombianas. Recuperado de Legiscomex.com.

Machado, A. (1999). La cuestión agraria y el desarrollo agropecuario. Cuadernos de Economía, 31, 237-279.

Morris, M., Schindehutte, M. \& Allen, J. (2005). The entrepreneur' business model: Toward a unified perspective. Journal of Business Review, 58, 726-735.

Ocati S.A. (2014). Ocati. Recuperado de www.ocati.com

Organización de las Naciones Unidas para la Agricultura y la Alimentación. (2002). El estado mundial de la agricultura y la alimentación 2002. Roma, Italia: Dirección de Información de la FAO. 
Portal Fruticola. (2010). Chile encabeza ranking de exportadoras de fruta. Recuperado de http://www.portalfruticola.com/2010/09/10/chile-dole-chile-encabeza-ranking-de-exportadoras-de-fruta/?pais=colombia

Portilla, B. (2000). La política agrícola en Chile: lecciones de tres décadas. Desarrollo Productivo, 68, 1-83.

Rendón, B., Rodríguez, J. \& Riascos, A. P. (2013). Análisis del impacto en el capital institucional de las cooperativas de ahorro y crédito frente a la aplicación de las NIIF. Caso cooperativas del Valle del Cauca. Cuadernos de Contabilidad, 14 (36), 881-901.

Socabio A.G. (2013, 11 de junio). Chile y Unifrutti se mantienen como líderes en exportación de frutas del país. Recuperado de http:// www.socabio.cl/index.php?option=com_content\&view=article\&i$d=5721$ :dole-chile-y-unifrutti-se-mantienen-como-lideres-en-exportacion-de-frutas-del-pais\&catid=36:noticias-socabio\&ltemid $=63$

Slywotzky, A. J. (1996). Value migration: how to think several moves ahead of the competition. Boston: Harvard Business Review Press.

Timmers, P. (1998). Business models for electronic markets. Electronic Markets, 8 (2), 3-8.

Yin, R. K. (2002). Case study reasearch: design and methods. London, UK: Sage Publications. 
Zerda, A. (2011). Colombia: del Japón de Suramérica a la confianza inversionista, dos estrategias para un patrón de crecimiento reprimarizante con iniquidad. Documentos FCE Escuela de Economía, 24, 18.

Zott, C., Amit, R. \& Massa, L. (2010). The business model: recent developments and futures researchs. Journal of Management, 37, 1-51. 